Posprint of: FEBS Letters Volume 585, Issue 19, 3 October 2011, Pages 2958-2964

\title{
A structural insight into the C-terminal RNA recognition motifs of T-cell intracellular antigen- 1 protein
}

Edited by Michael Ibba

Ángeles Aroca, Antonio Díaz-Quintana, Irene Díaz-Moreno

Instituto de Bioquímica Vegetal y Fotosíntesis, Universidad de Sevilla-CSIC, Avda. Américo Vespucio 49, Sevilla 41092, Spain

\begin{abstract}
T-cell intracellular antigen-1 (TIA-1) plays a pleiotropic role in cell homeostasis through the regulation of alternative pre-mRNA splicing and $m R N A$ translation by recognising uridine-rich sequences of RNAs. TIA-1 contains three RNA recognition motifs (RRMs) and a glutamine-rich domain. Here, we characterise its C-terminal RRM2 and RRM3 domains. Notably, RRM3 contains an extra novel $\mathrm{N}$-terminal $\alpha$-helix $(\alpha 1)$ which protects its single tryptophan from the solvent exposure, even in the two-domain RRM 23 context. The $\alpha 1$ hardly affects the thermal stability of RRM3. On the contrary, RRM2 destabilises RRM3, indicating that both modules are tumbling together, which may influence the RNA binding activity of TIA-1.
\end{abstract}

\section{Highlights}

- The extra N-terminal $\alpha$-helix of TIA-1 RRM3 is oriented in the two-domain RRM23 context.

- RRM3 is substantially destabilized by RRM2. TIA-1 RRM2 and RRM3 are tumbling together, with implications in RNA binding.

\section{Abbreviations}

$C D$, circular dichroism; $M D$, molecular dynamics; NMR, nuclear magnetic resonance; PRD, prion-related domain; RMSD, root mean square deviations; RMSF, root mean square fluctuations; RRM, RNA recognition motif; SG, stress granules; TEV, tobacco etch virus; TIA-1, T-cell intracellular antigen-1; XRD, X-ray diffraction

\section{Keywords}

DNA-RNA binding protein (D/RBP); RNA metabolism; RNA recognition motif (RRM); Proapoptotic protein; T-cell-restricted intracellular antigen-1 (TIA-1)

\section{Introduction}

Human T-cell intracellular antigen-1 (TIA-1) is a DNA/RNA binding protein (D/RBP) involved in transcriptional and post-transcriptional regulation of eukaryotic gene expression. TIA-1 modulates gene transcription rates in the nucleus by binding DNA [1] and [2] and regulating the alternative pre-mRNA splicing of ca. 15\% of human exons [3], [4], [5] and [6]. 
TIA-1 proteins at the cytoplasm control the turnover of mRNAs and/or repress their translation. Outstanding TIA-1 targets encode tumor necrosis factor alpha (TNF $\alpha$ ) and cytochrome c (Cc), among others [7], [8], [9], [10] and [11]. These mRNAs frequently bear adenine- and uridine-rich elements (AREs) in their $3^{\prime}$-untranslated regions ( $3^{\prime}$-UTRs) that bind trans-acting protein factors such as TIA-1 [10] and [12]. In response to environmental stress, TIA-1 promotes the assembly of a non-canonical preinitiation complex, in the cytoplasmic stress granules (SG), repressing the translation of these ARE-containing mRNAs [13] and [14]. In addition, the controlled aggregation of TIA-1 depends on the TDP-43 protein [15].

The role of TIA-1 in the control of cell death is of outstanding interest. In fact, TIA-1 seems to act as an apoptosis-promoting factor by controlling the alternative splicing of an mRNA encoding the membrane-bound form of the Fas receptor [6] and binding Cc-coding mRNA within SG, thereby precluding its degradation at processing bodies [9]. In fact, mice lacking TIA proteins show higher rates of embryonic lethality [16] cell proliferation and angiogenesis [17], suggesting that TIA-1 acts as a tumour suppressor.

TIA-1 is a ubiquitous 46-kDa multi-domain D/RBP that contains three RNA recognition motifs (RRMs), along with the prion-related domain (PRD), rich in glutamine, at the C-terminal end (Fig. S1 at Supplementary Data). Both PRD and the N-terminal RRM (RRM1) participate in the recruitment of U1snRNP [4]. Additionally, RRM1 associates with single-stranded DNA molecules (ssDNA) [1] but not with ssRNA [18]. In its turn, the second RRM domain (RRM2) is necessary for the RNA-binding activity of TIA-1 by the uridine-specific sequence recognition motif, whereas the C-terminal RRM domain (RRM3) binds weakly to ssRNA [18], [19] and [20].

RRM domains adopt a canonical $\beta 1 \alpha 1 \beta 2 \beta 3 \alpha 2 \beta 4$ topology and contain two well-conserved consensus sequences, named RNP2 ([I/L/V]-[F/Y]-[I/L/V]-X-N-L) and RNP1 ([K/R]-G-[F/Y]-[G/A]$[F / Y]-[I / L / V]-X-[F / Y])$, placed at $\beta 1$ and $\beta 3$ strands, respectively. The RNA binding site includes three aromatic side-chains located within these two RNPs. Canonical RRM domains use the $\beta$ sheet surface and one or two additional loops to bind RNA targets. However, several RRM-RNA complexes bind RNA or proteins in a different manner, owing to non-canonical secondary structure extensions in the $\mathrm{N}$ - or C-terminal domain [21]. Recently, a new type of RRMs named quasiRRMs (qRRMs) and identified in the hnRNP-F - shows a completely different mode for RNA recognition involving protein loops in RNA binding [22].

Given that TIA-1 is implicated in critical cellular events, exploring the domain rearrangement of TIA-1 protein along their biophysical properties would be highly valuable to understand the pleiotropic role of TIA-1 in the control of cell homeostasis. Notably, the RRM2 structure, as well as the identification of its RNA targets, has already been solved by NMR and XRD [23] and [24]. Within this frame, this work suggests that both RRM2 and RRM3 domains of TIA-1, which are connected by a 22-residue linker (Fig. S1 at Supplementary Data), do not behave independently in solution. Furthermore, RRM3 shows an extra N-terminal $\alpha$-helix, which protects its single tryptophan from the solvent exposure, even in the two-domain RRM23 unit context.

\section{Materials and methods}




\subsection{Site-directed mutagenesis of TIA-1 RRM constructs}

Isolated RRM2 and RRM3 modules, along with RRM23 - the two-domain in tandem - were constructed by PCR from the plasmid containing full-length TIA-1 [18], as described in Supplementary Data.

Design of the RRM2 mutants replacing tryptophans by phenylalanines, along with the RRM3$\Delta \alpha 1$ mutant, which lacks the $\mathrm{N}$-terminal $\alpha$-helix, was designed as explained in Supplementary Data.

\subsection{Protein expression and purification of TIA-1 RRM constructs}

Recombinant TIA-1 RRM domains, shown in Fig. S1 and previously cloned in pET11 vector, were expressed in E. coli BL21 (DE3) cells as followed in Supplementary Data.

Samples were concentrated to $0.1-0.4 \mathrm{mM}$ in $20 \mathrm{mM}$ potassium phosphate $(\mathrm{pH} 7.0)$ with 200 $\mathrm{mM} \mathrm{KCl}, 150 \mathrm{mM} \mathrm{MgCl} 2$ and $1 \mathrm{mM}$ DTT. Protein concentration was determined using spectrophotometry with predicted extinction coefficients. All molecular weights of the TIA-1 constructs used in this work were verified by MALDI-TOF spectroscopy.

\subsection{Circular dichroism spectroscopy}

All circular dichroism (CD) spectra were recorded on a Jasco J-815 spectropolarimeter, equipped with a Peltier temperature-control system, using a 1-mm quartz cuvette. The secondary structure analyses were carried out by recording their far-UV CD spectra (190-250 $\mathrm{nm}$ ) as explained in Supplementary Data.

Thermal unfolding was monitored between 280 and $373 \mathrm{~K}$ following a protocol described in Supplementary Data. For all these assays, the TIA-1 species at $10 \mu \mathrm{M}$ final concentration were solved into $20 \mathrm{mM}$ sodium phosphate $(\mathrm{pH} 7.0)$ with $5 \mathrm{mM} \mathrm{KCl}$ and $5 \mathrm{mM} \mathrm{MgCl}$.

\subsection{Fluorescence measurements}

Emission spectra were monitored using a Perkin-Elmer LS-5 fluorimeter equipped with a water-thermostat cell holder. Ten micromolar of isolated RRM2 and RRM3 and $10 \mu \mathrm{M}$ of RRM23 were solved into $20 \mathrm{mM}$ sodium phosphate ( $\mathrm{pH} 7.0)$ with $200 \mathrm{mM} \mathrm{KCl}, 150 \mathrm{mM} \mathrm{MgCl} 2$ and $1 \mathrm{mM}$ DTT. Then, they were incubated with increasing amounts of $\mathrm{GdnHCl}(1-6.5 \mathrm{M})$ concentration for $1 \mathrm{~h}$ at $293 \mathrm{~K}$ before recording the spectra (see Supplementary Data).

\subsection{Modelling and molecular dynamics}

Molecular dynamics (MD) computations were performed on the structure of the RRM3 domain of TIA-1 - along with the structure of the RRM3- $\Delta \alpha 1$ mutant - which was modelled as described in Supplementary Data.

Detailed MD protocol is explained in Supplementary Data.

\section{Results}




\subsection{TIA-1 RRM3 shows an extra $\mathrm{N}$-terminal $\alpha$-helix}

The crystallographic structure of the human TIA-1 RRM2 [24] shows a quasi-canonical RRM adopting the well-described $\beta \alpha \beta \beta \alpha \beta$ topology (Fig. S1 at Supplementary Data). However, it shows a new loop connecting two $\beta$-strands ( $\beta 2$ and $\beta 2^{\prime}$ ) that plays a role in binding uridinerich stretches [23].

We have obtained a homology model of the structure of human TIA-1 RRM3 (Fig. S1). Surprisingly, this model displays an extra, short $\alpha$-helix at its $\mathrm{N}$-terminal end, named $\alpha 1$. The sequence alignment of RRM3 from different species indicates that $\alpha 1$ is highly conserved among kingdoms (Fig. S1).

Our homology model is in a good agreement with the secondary structure contents for TIA-1 constructs. Fig. 1 shows the normalized far-UV CD spectra of RRM2, RRM3 and both C-terminal RRM domains in tandem (RRM23). Notably, the different domains only show minor differences in their global secondary structures, as summarised in Table S1 at Supplementary Data. In fact, RRM3 and RRM23 domains show helical compositions significantly larger than that of RRM2. This finding can be attributed to the trend of the $\mathrm{N}$-terminal amino acid sequence of RRM3 to form a helix. Such kind of re-arrangement may also be present in the two-domain construct.

To corroborate the amino-acid sequence involved in the $\mathrm{N}$-terminal $\alpha$-helix formation, the mutant RRM3- $\triangle \alpha 1$ was designed. Indeed, the deletion of three well-conserved residues placed at N-terminal $\alpha 1$-helix (Glu193-Val194-Val195 stretch) is sufficient to decrease the $\alpha$-helix content of RRM3 down to a value similar to that found for RRM2 (Table S1).

To test the structural model further, we subjected RRM3 and RRM3- $\triangle \alpha 1$ to MD calculations. Results are summarised in Fig. 2. At $298 \mathrm{~K}$, root mean square deviations (RMSD, Fig. S2) from the energy-minimised WT and $\Delta \alpha 1$ domain cores are $1.6 \AA$ and $1.3 \AA$, respectively. The largest atomic fluctuations - measured as root mean square fluctuations (RMSF) - map at the end terminus linker, loop regions within the RRM domain, and the C-terminus (Fig. 2A). Notably, $\alpha 1$ helix remains unaltered and keeps its position along the trajectories reckoned at 298 and $398 \mathrm{~K}$ (Fig. 2B). Analysis of the trajectories showed that strand $\beta 4$, which lies near $\alpha 1$, is the less stable regular element of the domain. In fact, this strand is lost during the simulations performed at $398 \mathrm{~K}$ (Fig. 2B).

\subsection{TIA-1 RRM3 is destabilised by RRM2}

Given that TIA-1 RRM3 is extended by helix $\alpha 1$ at its N-terminus (Fig. S1 and Table S1), we tested whether this fact affects the thermal stability of the domain. CD spectroscopy indicates that the loss of $\alpha 1$ has no consequences on protein thermal stability. Actually, RRM 3 and RRM3- $\Delta \alpha 1$ show identical values for the midpoint melting temperature (Tm) (Fig. 3A and B).

Interactions between neighbouring RNA binding domains within an RBP influence their structure and stability [25]. To test a putative interaction between RRM3 and the adjacent RRM2 of TIA-1, we have performed thermal unfolding studies on the single modules and the two-domain construct. CD data shows that the Tm for isolated RRM2 (333.5 $\pm 1.05 \mathrm{~K}$; Fig. $3 \mathrm{C}$ ) is substantially lower than the one for RRM3 (Fig. 3A or 3B). Notably, the unfolding curve for RRM23 (326.8 $\pm 0.4 \mathrm{~K}$; Fig. 3D) cannot be reproduced by lineal combination of the curves for 
the individual domains, as might be in the absence of interactions between them. In fact, the respective Tm values of RRM2 and RRM3 are ca. 6 and $22 \mathrm{~K}$ higher than that of RRM23. This clearly indicates that inter-domain interactions are taking place [25]. Intriguingly, such an interaction substantially destabilises RRM3.

\subsection{The $\mathrm{N}$-terminal $\alpha$-helix of TIA-1 RRM3 partially protects its tryptophan from exposure to solvent in the presence of RRM2}

Fig. 4A shows the fluorescence emission spectra of TIA-1 constructs. Whereas RRM2 and two C-terminal domains in tandem RRM23 have the maximum fluorescence intensity at 354 and $352 \mathrm{~nm}$ respectively, the isolated RRM3 shows a maximum at $308 \mathrm{~nm}$. This indicates that tryptophan residues are well-exposed to the aqueous solvent in the native structure of RRM2 and RRM23, which have four and five tryptophans, respectively. However, the single tryptophan placed at RRM3 - Trp272 - was buried in the protein core. These results are consistent with the human TIA-1 RRM2 structure [23] and the homology model built for TIA-1 RRM3 herein proposed. Actually, Fig. S1 shows how Trp272 anchors the end of strand $\beta 4$ to the domain core formed by helices $\alpha 1$ and $\alpha 3$.

For the RRM3- $\Delta \alpha 1$ mutant, the fluorescence maximum shifts to $325 \mathrm{~nm}$, indicating that $\alpha 1$ is partially occluding Trp272 (Fig. 4A). Although Trp272 is partially buried in both, RRM3 and RRM3- $\Delta \alpha 1$, the aromatic residue is slightly more solvated in RRM3- $\Delta \alpha 1$ mutant than in the wild-type species. In fact, the side-chain of this residue shows $8.9 \AA 2$ exposed surface in RRM3, whereas it increases up to $11.8 \AA 2$ in the RRM3- $\Delta \alpha 1$. Fig. $2 \mathrm{C}$ displays the radial distribution functions of water molecules around Trp272. A well-structured first solvation sphere is observed for this residue in RRM3 and RRM3- $\Delta \alpha 1$ structures, containing, on average, 3.2 and 4.7 water molecules, respectively.

On the other hand, GndCl-induced transition curves of the RRM3 and the mutant RRM3- $\Delta \alpha 1$ show no differences in the midpoint $\mathrm{GndCl}$ concentration $(\mathrm{Cm})$ of unfolding transitions, by recording the fluorescence intensity at $350 \mathrm{~nm}$ (Fig. 4B and C). Actually, $\mathrm{Cm}$ values are identical: $4.54 \mathrm{M} \pm 0.02 \mathrm{M}$ and $4.52 \pm 0.02 \mathrm{M}$ for RRM3 and RRM3- $\Delta \alpha 1$ species, respectively.

To corroborate whether the relative orientation between the additional $\alpha$-helix of RRM 3 and Trp272 remains unchanged in the two-domain construct, we designed an RRM23 mutant in which four out of its five tryptophans - Trp80, Trp147, Trp160 and Trp170 - were replaced by phenylalanines, following the same strategy as before [26]. Consequently, only Trp272 located at RRM3 remains at the RRM23 mutant, named RRM23-Trp272. As a result, the fluorescence emission spectrum for the RRM23-Trp272 almost overlaps with that of the RRM3 (Fig. 4A). In fact, both the 308-nm maximum and the intensity in fluorescence signal of RRM23-Trp272 are close to the ones observed for the isolated RRM3. This indicates that Trp272 remains buried into the core of RRM23 construct as part of the TIA-1 protein. Interestingly, tryptophan-byphenylalanine substitutions present in RRM23-Trp272 mutant have no significant effects on its secondary structure with regard the RRM23 (Table S1). Moreover, the RRM23-Trp272 mutant behaves as RRM23 in terms of stability with a Tm value of $325.1 \pm 2.4 \mathrm{~K}$ (Fig. 3D and E). 
As expected from a two-domain construct, chemical denaturalization performed by the addition of increasing $\mathrm{GndCl}$ concentration (1-6.5 M) results in a sigmoidal transition curve with a $\mathrm{Cm}$ significantly larger than that observed for the single RRM3 $(5.50 \pm 0.01 \mathrm{M})$, suggesting that tandem domains are more resistant to denaturation by chemical agents.

\section{Discussion}

Our results suggest the presence of an extra $\mathrm{N}$-terminal $\alpha$-helix - named $\alpha 1$ - as part of the RRM3 domain of TIA-1. This $\alpha 1$ at the $\mathrm{N}$-end is surprisingly well-conserved among kingdoms, so it might be performing a common function. $\alpha 1$-extended RRM3 does not match with the canonical topology reported for classical RRMs although changes in the number and order of the secondary structure elements in RRM domains have been extensively reported. For instance, the C-terminal RRM of La and U1A snRNP proteins is extended by an $\alpha$-helix that lies on the $\beta$-sheet surface [27] and [28]. Similarly it happens for the CstF-64 protein, which also has another short $\alpha$-helix at the $\mathrm{N}$-terminus, in addition to that at the $\mathrm{C}$-terminal end [29]. Noteworthy, this helix is adjacent to the first $\beta$ strand so it shows a different orientation in comparison to that of $\alpha 1$ in TIA-1 RRM3. In addition, the middle RRM domains of PTB protein show a $\beta$-sheet constituted by five $\beta$-strands [30]. These changes in the RRM topology are involved in RNA-protein and/or protein-protein interactions.

Of interest is the role that this novel helix $\alpha 1$ of RRM3 plays in the context of the full-length TIA-1 protein. Thermal denaturation studies on RRM3 wild-type and on the mutant lacking the helix $\alpha 1$ (RRM3- $\Delta \alpha 1$ ) reveal that $\alpha 1$ is not clue for protein stability. Moreover, Trp272 placed at RRM3- $\beta 4$ seems to be partially buried by the protein core - mainly by $\alpha 3$ - as well as by $\alpha 1$, as shown in Fig. 5A. Indeed, when RRM3 looses $\alpha 1$, the fluorescence measurements and MD simulations indicate that Trp272 is less protected from solvent exposure. It is worth to mention that the relative orientation between $\alpha 1$ and the domain core containing Trp272 remains unchanged in the two-domain RRM23 construct, as the fluorescence spectrum of RRM23-Trp272 almost overlaps with that of RRM3.

The presence of hydrophobic interactions between amino acids pairs Leu189-Leu255 and Val194-Ala252 (Fig. 5A), which are burying Trp272 of exposed surface, seems to stabilise the interaction among the secondary structure elements $\alpha 1, \alpha 3$ and $\beta 4$. Actually, $\alpha 1$ is wellcompacted onto the domain core, as inferred from the space-filling representation (Fig. 5B). Recent structural studies show that not only the $\beta$-sheet surface but also the loops and other extra non-canonical secondary elements can be crucial for nucleic acid or protein recognition [23]. The human U11/U12-65 K protein of the spliceosome contains an extension in the $\mathrm{N}$ terminal tail which supports the appropriate folding of the protein and orientation of RNAbinding elements [31]. Within this frame, the orientation of the well-conserved extra helix $\alpha 1$ of RRM3 in the full-length TIA-1 context and the fact that Trp272 has remained unchanged from kingdoms during evolution could play the key to understand the role of TIA-1 in the RNA/protein recognition events.

The analysis of RRM structures solved to date shows that two consecutive RRMs separated by a linker can interact with each other forming a compact unit, which can be induced by the presence of RNA/DNA, but also occurs in the absence of it [32]. In this study, the thermal unfolding analyses reveal that RRM3 is the most stable RRM domain of TIA-1 protein. The 
unfolding studies of isolated C-terminal RRM domains and the tandem RRM23 have revealed substantial differences in terms of thermal stability of RRM3 when RRM2 is part of the same construct. In fact, RRM3 decreases its thermal stability in ca. $20 \mathrm{~K}$ in the RRM23 tandem. These data help to explain that both single domains RRM2 and RRM3 of human TIA-1 do not operate independently in solution. Therefore, it is tempting to speculate that this modular interaction between RRM2 and RRM3 might be focused in the RNA binding activity of TIA-1 similarly to what previously observed for RRM3-RRM4 domains of PTB protein [32] and the two Nterminal RRM domains of the homologous yeast Pub1 protein [33], in order to create a highaffinity RNA-binding unit. Lastly, contacts between RRM2 and RRM3 in TIA-1 may be key to stabilise a suitable conformation that can adapt to the changes in the direction of the RNA chain inside the highly structured $5^{\prime}$ splice site of exon 6 of RNA encoding the Fas receptor and/or 3'-UTRs, as was proposed for the central K-Homology domains of KH-type splicing regulatory protein (KSRP) [25]. Still, additional structural studies will help us to understand the properties of the novel extra helix $\alpha 1$ in RRM3 and the rearrangement between both Cterminal RRM domains of TIA-1 within RNA/DNA binding context.

\section{Acknowledgements}

The authors wish to thank the Andalusian Government (P07-CVI-02896 and BIO198) for financial support. The plasmid containing the TIA-1 full-length protein was kindly provided by Dr. M. Gorospe (National Institutes of Health, Baltimore, USA) and Dr. P. Anderson (Harvard Medical School, Boston, USA). We are grateful to Prof. Miguel A. De la Rosa for critical reading of the manuscript. 


\section{References}

[1] E.A. Suswam, Y. Li, H. Mahtani, P.H. King

Novel DNA-binding properties of the RNA-binding protein TIAR

Nucl. Acids Res., 33 (2005), pp. 4507-4518

[2] A. McAlinden, L. Liang, Y. Mukudai, T. Imamura, L.J. Sandell

Nuclear protein TIA-1 regulates COL2A1 alternative splicing and interacts with precursor mRNA and genomic DNA

J. Biol. Chem., 282 (2007), pp. 24444-24454

[3] P. Förch, O. Puig, N. Kedersha, C. Martínez, S. Granneman, B. Séraphin, P. Anderson, J. Valcárcel

The Apoptosis-Promoting Factor TIA-1 is a regulator of alternative pre-mRNA splicing

Mol. Cell, 6 (2000), pp. 1089-1098

[4] P. Forch, O. Puig, C. Martinez, B. Seraphin, J. Valcarcel

The splicing regulator TIA-1 interacts with U1-C to promote U1 snRNP recruitment to $5^{\prime}$ splice sites

EMBO J., 21 (2002), pp. 6882-6892

[5] J.M. Izquierdo, N. Majós, S. Bonnal, C. Martínez, R. Castelo, R. Guigó, D. Bilbao, J. Valcárcel

Regulation of Fas alternative splicing by antagonistic effects of TIA-1 and PTB on exon definition

Mol. Cell, 19 (2005), pp. 475-484

[6] J.M. Izquierdo

Heterogeneous ribonucleoprotein C displays a repressor activity mediated by T-cell intracellular antigen-1-related/like protein to modulate Fas exon 6 splicing through a mechanism involving $\mathrm{Hu}$ antigen $\mathrm{R}$

Nucl. Acids Res., 38 (2010), pp. 8001-8014

[7] C. Gueydan, L. Droogmans, P. Chalon, G. Huez, D. Caput, V.r. Kruys

Identification of TIAR as a protein binding to the translational regulatory AU-rich element of TNF- $\alpha$ mRNA

J. Biol. Chem., 274 (1999), pp. 2322-2326

[8] M. Piecyk, S. Wax, A.R.P. Beck, N. Kedersha, M. Gupta, B. Maritim, S. Chen, C. Gueydan, V. Kruys, M. Streuli, P. Anderson 
TIA-1 is a translational silencer that selectively regulates the expression of TNF- $\alpha$

EMBO J., 19 (2000), pp. 4154-4163

[9] T. Kawai, A. Lal, X. Yang, S. Galban, K. Mazan-Mamczarz, M. Gorospe

Translational control of cytochrome $\mathrm{c}$ by RNA-binding proteins TIA-1 and HuR

Mol. Cell. Biol., 26 (2006), pp. 3295-3307

[10] I. Lopez de Silanes, S. Galban, J.L. Martindale, X. Yang, K. Mazan-Mamczarz, F.E. Indig, G. Falco, M. Zhan, M. Gorospe

Identification and functional outcome of mRNAs associated with RNA-binding protein TIA-1

Mol. Cell. Biol., 25 (2005), pp. 9520-9531

[11] K. Mazan-Mamczarz, A. Lal, J.L. Martindale, T. Kawai, M. Gorospe

Translational repression by RNA-binding protein TIAR

Mol. Cell. Biol., 26 (2006), pp. 2716-2727

[12] S. Yamasaki, G. Stoecklin, N. Kedersha, M. Simarro, P. Anderson

T-cell intracellular antigen-1 (TIA-1)-induced translational silencing promotes the decay of selected mRNAs

J. Biol. Chem., 282 (2007), pp. 30070-30077

[13] N. Kedersha, M. Gupta, W. Li, I. Miller, P. Anderson

RNA-binding proteins TIA-1 and TIAR link the phosphorylation of elF-2 alpha to the assembly of mammalian stress granules

J. Cell. Biol., 147 (1999), pp. 1431-1442

[14] N. Kedersha, G. Stoecklin, M. Ayodele, P. Yacono, J.E. Lykke-Andersen, M.J. Fritzler, D. Scheuner, R.J. Kaufman, D.E. Golan, P. Anderson

Stress granules and processing bodies are dynamically linked sites of mRNP remodeling

J. Cell. Biol., 169 (2005), pp. 871-884

[15] K.K. McDonald, A.s. Aulas, L. Destroismaisons, S. Pickles, E. Beleac, W. Camu, G.A. Rouleau, C. Vande Velde

TAR DNA-Binding Protein 43 (TDP-43) regulates stress granule dynamics via differential regulation of G3BP and TIA-1

Hum. Mol. Genet., 20 (2011), pp. 1400-1410

[16] A.R.P. Beck, I.J. Miller, P. Anderson, M. Streuli 
RNA-binding protein TIAR is essential for primordial germ cell development

Proc. Natl. Acad. Sci. USA, 95 (1998), pp. 2331-2336

[17] J.M. Izquierdo, J. Alcalde, I. Carrascoso, R. Reyes, M.D. Ludeña

Knockdown of T-cell intracellular antigens triggers cell proliferation, invasion and tumour growth

Biochem. J., 435 (2011), pp. 337-344

[18] L.M. Dember, N.D. Kim, K.Q. Liu, P. Anderson

Individual RNA recognition motifs of TIA-1 and TIAR have different RNA binding specificities

J. Biol. Chem., 271 (1996), pp. 2783-2788

[19] H.S. Kim, Y. Kuwano, M. Zhan, R. Pullmann Jr., K. Mazan-Mamczarz, H. Li, N. Kedersha, P. Anderson, M.C.J. Wilce, M. Gorospe, J.A. Wilce

Elucidation of a C-rich signature motif in target mRNAs of RNA-binding protein TIAR

Mol. Cell. Biol., 27 (2007), pp. 6806-6817

[20] H.S. Kim, M.C.J. Wilce, Y.M.K. Yoga, N.R. Pendini, M.J. Gunzburg, N.P. Cowieson, G.M. Wilson, B.R.G. Williams, M. Gorospe, J.A. Wilce

Different modes of interaction by TIAR and HuR with target RNA and DNA

Nucl. Acids Res., 39 (2011), pp. 1117-1130

[21] A. Cléry, M. Blatter, F.H.T. Allain

RNA recognition motifs: boring? Not quite

Curr. Opin. Struct. Biol., 18 (2008), pp. 290-298

[22] C. Dominguez, J.F. Fisette, B. Chabot, F.H.T. Allain

Structural basis of G-tract recognition and encaging by hnRNP F quasi-RRMs

Nat. Struct. Mol. Biol., 17 (2010), pp. 853-861

[23] K. Kuwasako, M. Takahashi, N. Tochio, C. Abe, K. Tsuda, M. Inoue, T. Terada, M. Shirouzu, N. Kobayashi, T. Kigawa, S. Taguchi, A. Tanaka, Y. Hayashizaki, P. Güntert, Y. Muto, S. Yokoyama

Solution structure of the second RNA Recognition Motif (RRM) domain of murine T-cell intracellular antigen-1 (TIA-1) and its RNA recognition mode

Biochemistry, 47 (2008), pp. 6437-6450

[24] A.O. Kumar, M.C. Swenson, M.M. Benning, C.L. Kielkopf 
Structure of the central RNA recognition motif of human TIA-1 at $1.95 \AA$ resolution

Biochem. Biophys. Res. Comm., 367 (2008), pp. 813-819

[25] I. Díaz-Moreno, D. Hollingworth, G. Kelly, S. Martin, M. García-Mayoral, P. Briata, R. Gherzi, A. Ramos

Orientation of the central domains of KSRP and its implications for the interaction with the RNA targets

Nucl. Acids Res., 38 (2010), pp. 5193-5205

[26] J.M. García-Heredia, I. Díaz-Moreno, P.M. Nieto, M. Orzáez, S. Kocanis, M. Teixeira, E. Pérez-Payá, A. Díaz-Quintana, M.A. De la Rosa

Nitration of tyrosine 74 prevents human cytochrome $\mathrm{c}$ to play a key role in apoptosis signaling by blocking caspase- 9 activation

Biochim. Biophys. Acta, 1797 (2010), pp. 981-993

[27] A. Jacks, J. Babon, G. Kelly, I. Manolaridis, P. Cary, S. Curry, M. Conte

Structure of the C-terminal domain of human La protein reveals a novel RNA recognition motif coupled to a helical nuclear retention element

Structure, 11 (2003), pp. 833-843

[28] J. Avis, F. Allain, P. Howe, G. Varani, K. Nagi, D. Neuhaus

Solution structure of the $\mathrm{N}$-terminal RNP domain of U1A protein: the role of C-terminal residues in structure stability and RNA binding

J. Mol. Biol., 257 (1996), pp. 398-411

[29] J.M. Perez-Canadillas, G. Varani

Recognition of GU-rich polyadenylation regulatory elements by human CstF-64 protein

EMBO J., 22 (2003), pp. 2821-2830

[30] M. Conte, T. Grune, J. Ghuman, G. Kelly, A. Ladas, S. Matthews, S. Curry

Structure of tandem RNA recognition motifs from polypyrimidine tract binding protein reveals novel features of the RRM fold

EMBO J., 19 (2000), pp. 3132-3141

[31] C. Netter, G. Weber, H. Benecke, M.C. Wahl

Functional stabilization of an RNA recognition motif by a non-canonical $\mathrm{N}$-terminal expansion

RNA, 15 (2009), pp. 1305-1313 
[32] F. Vitali, A. Henning, F.C. Oberstrass, Y. Hargous, S.D. Auweter, M. Erat, F.H.T. Allain

Structure of the two most C-terminal RNA recognition motifs of PTB using segmental isotope labeling

EMBO J., 25 (2006), pp. 150-162

[33] H. Li, H. Shi, H. Wang, Z. Zhu, X. Li, Y. Gao, Y. Cui, L. Niu, M. Teng

Crystal structure of the two N-terminal RRM domains of Pub1 and the poly (U)-binding properties of Pub1

J. Struct. Biol., 171 (2010), pp. 291-297 


\section{Figure captions}

Figure 1. Far-UV (190-250 nm) CD spectra of different TIA-1 domain constructs. RRM domains are represented as follows: RRM2 in dotted line, RRM3 in solid line and RRM23 in dashed line.

Figure 2. MD calculations. (A) Diagram of RRM3 average structure. Worm radius is proportional to atomic fluctuations (RMSF). RMSF values lower than $0.7 \AA$ are coloured from yellow to orange, and those between 0.7 and $6.0 \AA$ are coloured from orange to red. (B) Secondary structure timelines for RRM3 (left) and RRM3- $\Delta \alpha 1$ (right) at $298 \mathrm{~K}$ (upper) and $398 \mathrm{~K}$ (lower). Turns are in gold yellow, $\alpha$-helices in red, $\pi$-helices in orange, 310 helices in pink and $\beta$-bridges are in blue. (C) Radial distribution functions of water molecules around Trp272.

Figure 3. Far-UV $230 \mathrm{~nm}-\mathrm{CD}$ signal of RRM3 (A), RRM3- $\Delta \alpha 1$ (B), RRM2 (C), RRM23 (D) and RRM23-Trp272 (E) recorded during thermal denaturation experiments. The fitted curves of unfolding are represented by continuous lines, and they are superimposed on experimental data (black dots). Transition midpoints are marked by dashed lines.

Figure 4. Fluorescence measurements of TIA-1 constructs (A) Fluorescence intensity of native TIA-1 domain constructs: RRM2 (- - - ; dash dot dot line), RRM23-WT (- - -; dash dot line), RRM3 (.$\cdots$; dotted line), RRM3- $\Delta \alpha 1$ (- - -; dashed line) and RRM23-Trp272 (-; solid line). (B-D) Guanidine chloride-induced unfolding curves of RRM3 (B), RRM3- $\triangle \alpha 1$ (C) and RRM23Trp272 (D) by recording changes at the 350-nm fluorescence signal. The fitted unfolding curves are represented in solid line, and they are superimposed on experimental data (black dots). The denaturant concentration at half-completion of the transition is marked by dashed lines.

Figure 5. Close-up of the TIA-1 RRM3 structural model. (A) The classical RRM fold is in orange highlighting $\alpha 1$ in blue and $\alpha 3$, in green. Side-chains of Gln197, Ser198, Cys203, His248 and Trp272 are represented in CPK coloured-code. Hydrophobic interactions between amino acids pairs Leu189-Leu255 and Val194-Ala252 are shown in magenta and yellow, respectively. (B) Space-filling representation of the TIA-1 RRM3 is shown in the same orientation as on (A) and $180^{\circ}$-rotated. The same coloured-code than in $(A)$ is used for the secondary structure elements. Trp272 is shown in grey. 
Figure 1

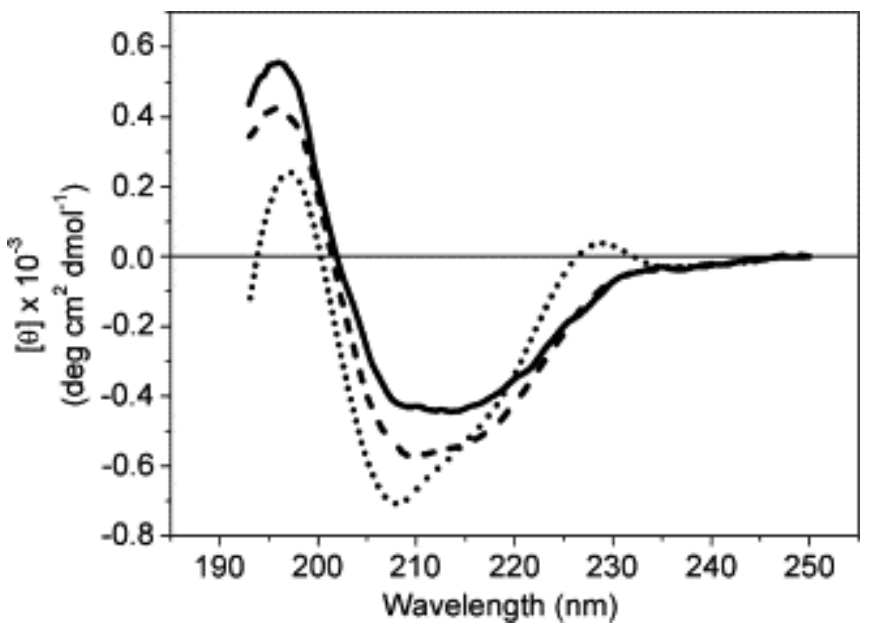


Figure 2

A

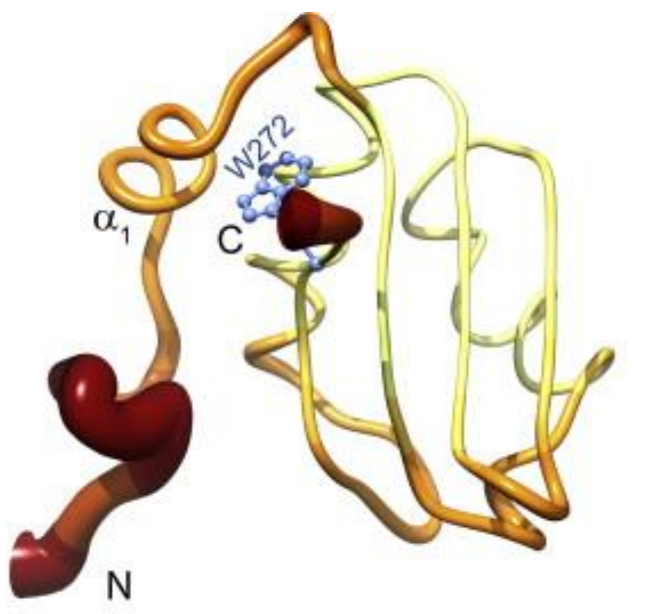

B

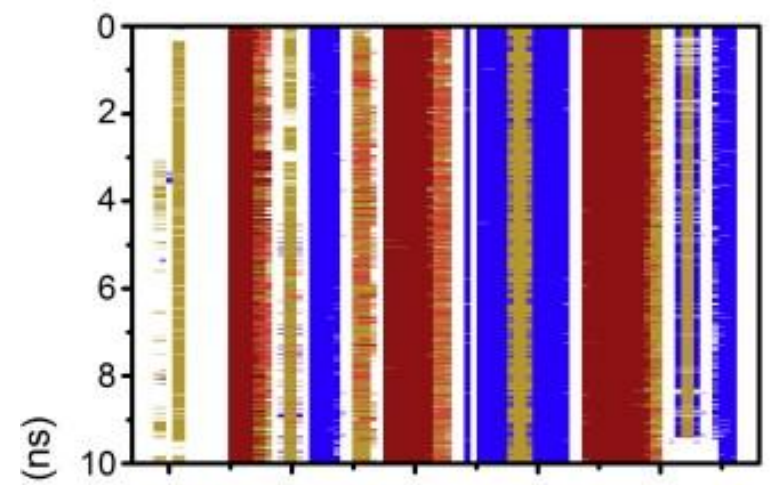

$\stackrel{\oplus}{E}$
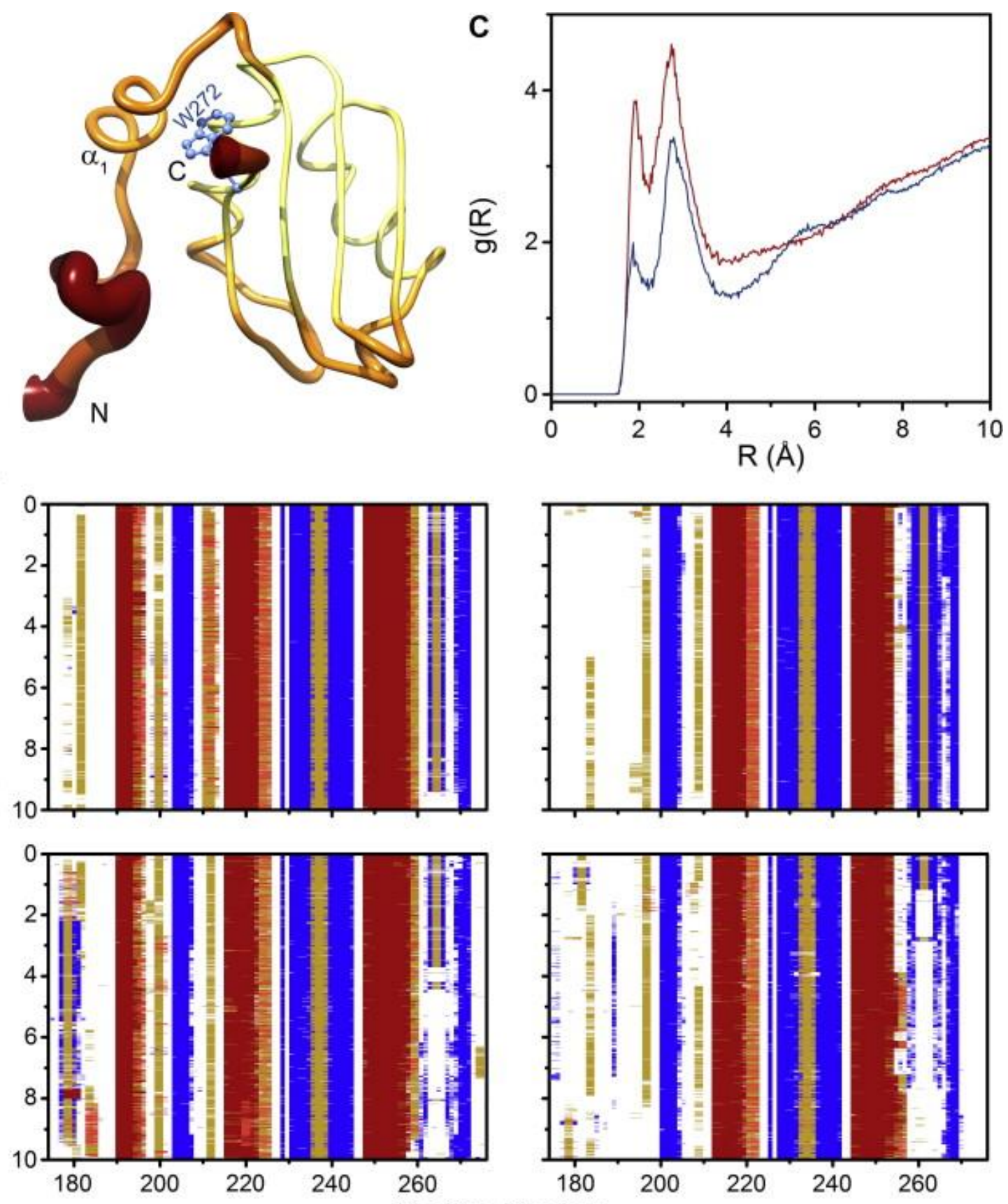
Figure 3

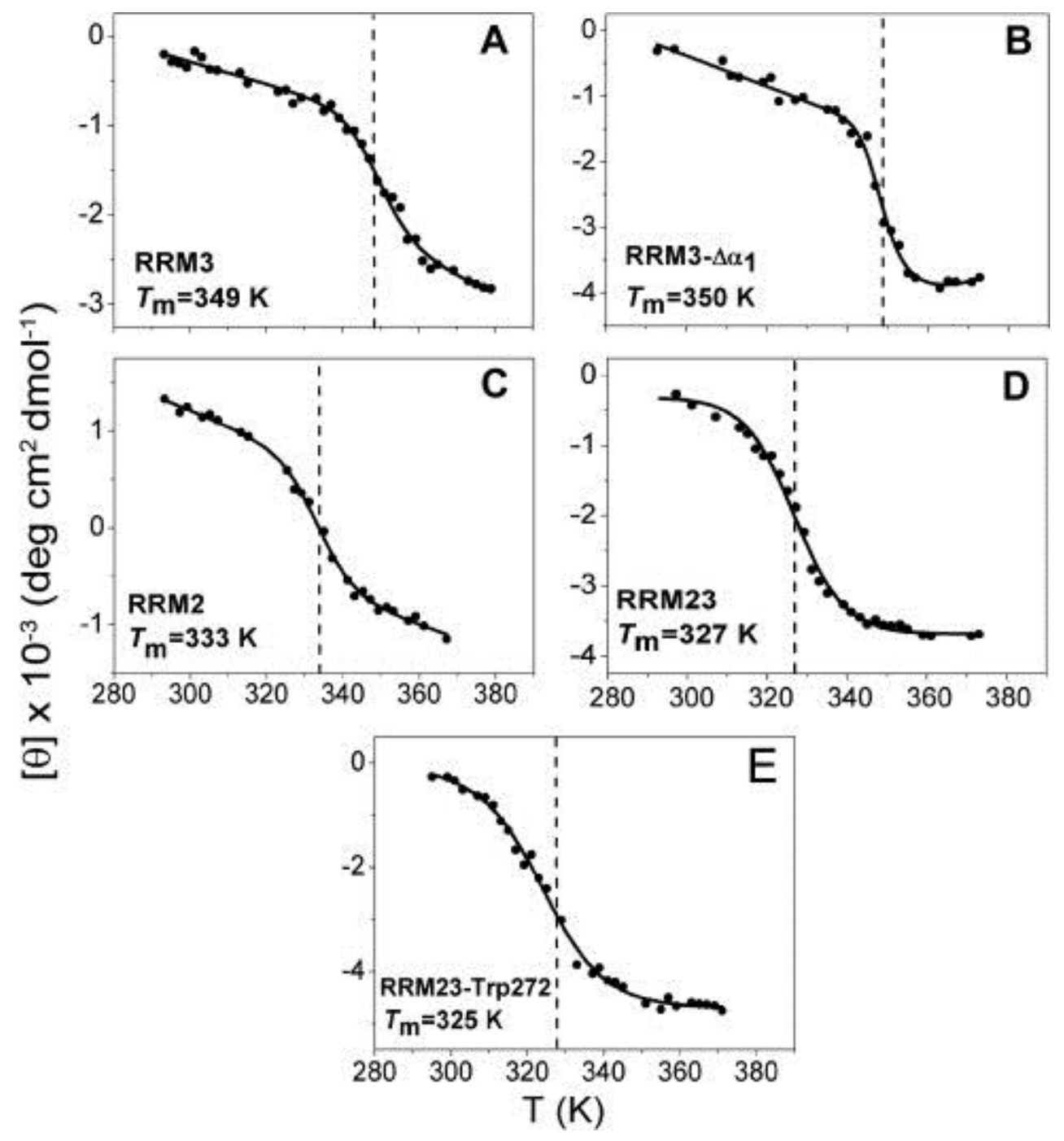


Figure 4
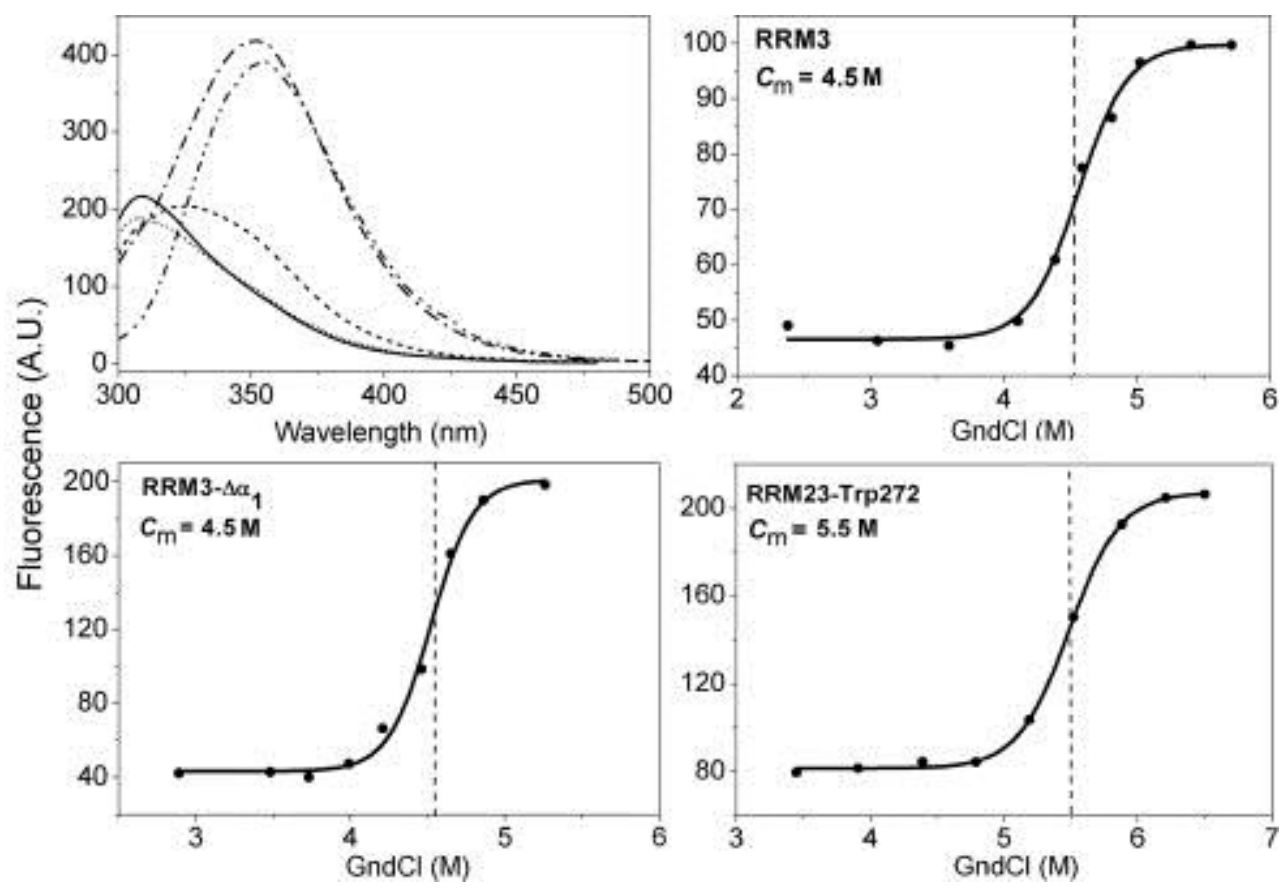
Figure 5

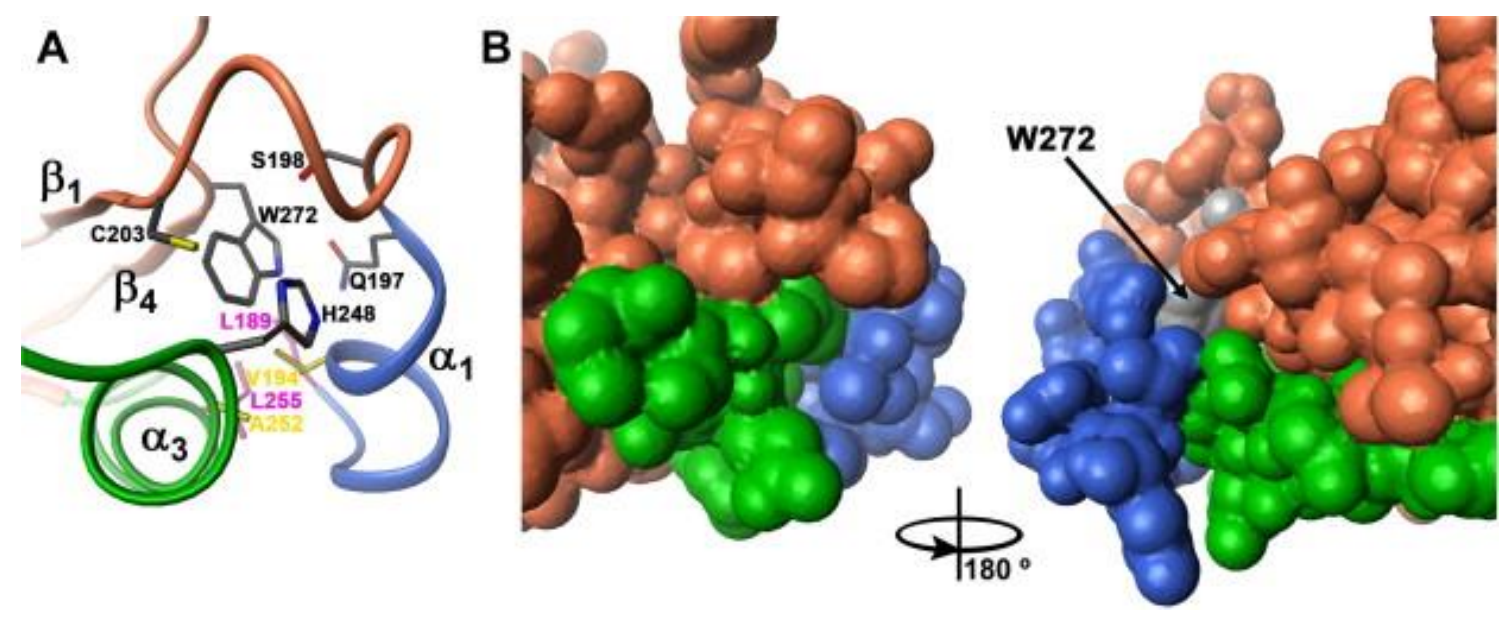

